DFTT 30/96

NORDITA 96/37P

\title{
A solvable twisted one-plaquette model
}

\author{
M. Billóq日 \\ Nordita, Blegdamsvej 17, Copenhagen Ø, Denmark \\ A. D'Adda \\ Istituto Nazionale di Fisica Nucleare, Sezione di Torino and Dipartimento di Fisica \\ Teorica dell'Università di Torino, via P.Giuria 1, I-10125 Turin,Italy
}

\begin{abstract}
We solve a hot twisted Eguchi-Kawai model with only timelike plaquettes in the deconfined phase, by computing the quadratic quantum fluctuations around the classical vacuum. The solution of the model has some novel features: the eigenvalues of the time-like link variable are separated in $L$ bunches, if $L$ is the number of links of the original lattice in the time direction, and each bunch obeys a Wigner semicircular distribution of eigenvalues. This solution becomes unstable at a critical value of the coupling constant, where it is argued that a condensation of classical solutions takes place. This can be inferred by comparison with the heat-kernel model in the hamiltonian limit, and the related Douglas-Kazakov phase transition in QCD2. As a byproduct of our solution, we can reproduce the dependence of the coupling constant from the parameter describing the asymmetry of the lattice, in agreement with previous results by Karsch.
\end{abstract}

\section{Introduction}

The twisted one-plaquette models, or Eguchi-Kawai models [1, 2], have been extensively studied in the last fifteen years for their remarkable property of satisfying, in the large $N$ limit, the same loop equations as the corresponding lattice gauge theories. The reduction of the space-time degrees of freedom to internal degrees of freedom is the most startling property of these models. Their solution would amount to finding the master field for lattice gauge theories, and to solving them

\footnotetext{
${ }^{a}$ Supported by the Istituto Nazionale di Fisica Nucleare, Italy

${ }^{b}$ e-mail: billo@alf.nbi.dk

${ }^{c}$ e-mail: dadda@to.infn.it
} 
in the large $N$ limit. Unfortunately, in spite of the reduction to one plaquette, the saddle point structure of the Eguchi-Kawai model in quite complicated, due to the effect of the twists, and a complete solution appears to be beyond the reach of our present techniques.

In this paper we study a twisted one-plaquette model, which is much simpler than the complete twisted Eguchi-Kawai model, but whose solution can be explicitly found, at least in the weak coupling (or high temperature) regime, in terms of eigenvalue distribution. The model under consideration is defined by the partition function

$$
Z=\int D V \int \prod_{\mu=1}^{d} D U_{\mu} \mathrm{e}^{\beta N \operatorname{Re}^{\mathrm{i} \frac{2 \pi}{L}} \sum_{\mu=1}^{d} \operatorname{Tr}\left(V U_{\mu} V^{\dagger} U_{\mu}^{\dagger}\right)}
$$

and in the large $N$ limit it is equivalent, as shown later in the paper, to a $d+1$ dimensional lattice gauge theory at finite temperature, with $L$ links in the compactified time dimension, and with only the couplings induced by time-like plaquettes. Hence the action described by eq. (1) arises naturally in finite temperature lattice gauge theories as a zero order approximation in which space-like plaquettes are completely neglected. This approximation has already been discussed in previous papers [3, 4] and it will be reviewed in the following section.

The main result of our paper consists in solving the model in the broken phase, which occurs for values of $\beta$ larger than a critical value $\beta_{\mathrm{cr}}$ and is characterised by a non vanishing vacuum expectation value of the Polyakov loop $\mathcal{P}=\operatorname{Tr} V^{L}$. The solution has some novel, interesting features, namely an eigenvalue distribution for the matrix $V$ which consists of $L$ separated bunches centered around the $L^{\text {th }}$ roots of unity. The eigenvalue distribution within each bunch is given, with very good approximation, by a semicircular Wigner distribution of calculable radius. The splitting of the eigenvalue distribution into several bunches is a new feature that makes the model in consideration interesting in itself and susceptible of applications in other branches of physics, possibly for instance condensed matter physics.

The paper is organised as follows. In section 2 the twisted Eguchi-Kawai reduction in $3+1$ dimensions is considered in the framework of finite-temperature lattice gauge theories, and a scheme of dimensional reduction is proposed that allows to extend the analysis to $d+1$ dimensions. In section 3 we derive the effective model for the Polyakov loops in the deconfined phase, up to quadratic order in the fluctuations. In section 4 the solution of the effective model and the phase transition occurring at a critical value of the coupling are discussed for a model with arbitrary number $L$ of lattice links in the time direction. In this context we also derive the rescaling of the coupling $\beta$ with the parameter $\rho$, describing the asymmetry of the original lattice, and we find results in excellent agreement with the ones by Karsch in [5]. In section 5 we present our conclusions. 


\section{Dimensional reduction in finite temperature large $N$ LGT}

\subsection{Twisted reduction in $3+1$ dimensions}

The symmetric twisted Eguchi-Kawai model (TEK ) in $3+1$ dimensions is a one plaquette model which is equivalent, in the large $N$ limit, to a lattice $\mathrm{SU}(\mathrm{N})$ pure gauge theory defined in a periodic box of size $L$, with $N=L^{2}$. Its action is given by":

$$
S_{\text {sTEK }}=N \beta \sum_{\mu>\nu} \operatorname{Re} \operatorname{Tr}\left(\mathrm{e}^{\mathrm{i} \frac{2 \pi}{L}} U_{\mu} U_{\nu} U_{\mu}^{\dagger} U_{\nu}^{\dagger}\right) .
$$

In the large $N$ limit the size of the box goes to infinity in all directions. If we want to describe a finite temperature gauge theory we need a lattice whose size in the compactified time direction remains finite also in the large $N$ limit. This presents a problem in the TEK approach where the size of the box is linked to $N$. The problem can be overcome in two different ways. We can define the theory in an asymmetric box, whose spatial extent goes to infinity in the large $N$ limit while its extent in the time direction remains finite. Several of such boxes with the corresponding twists exist in the literature [6, 7]. On the other hand, we can keep a symmetric box of size $L$ but with different lattice spacings $a$ and $a_{\tau}$ respectively in space and time directions. If $T$ is the temperature, then $1 / T$ is the size of compactified time dimension, given by:

$$
\frac{1}{T}=L a_{\tau}
$$

Let us introduce the asymmetry parameter $\rho=a / a_{\tau}$. Then from (3) we have

$$
\rho=a T L .
$$

From eq. (4) it is clear that in order to keep $T$ finite the parameter $\rho$ has to go to infinity like $L=N^{1 / 2}$ in the large $N$ limit. The introduction of different lattice spacings is equivalent to the introduction of different couplings $\beta_{s}$ and $\beta_{t}$ for spacelike and time-like plaquettes respectively. The relation between these new couplings and the asymmetry parameter $\rho$ is given by

$$
\beta_{t}(\rho)=\rho\left(\beta_{\mathrm{symm}}+4 c_{\tau}(\rho)\right) \quad ; \quad \beta_{s}(\rho)=\frac{\left(\beta_{\mathrm{symm}}+4 c_{\sigma}(\rho)\right)}{\rho},
$$

where $\beta_{\text {symm }}$ is the coupling corresponding to a symmetric lattice, namely to $\rho=1$. The terms proportional to $\beta_{\text {symm }}$ in (5) stem from the requirement that a YangMills theory is reproduced in the naive or classical continuum limit. The functions $c_{\tau}$ and $c_{\sigma}$ on the other hand represent the result of quantum corrections and have

\footnotetext{
${ }^{1}$ In this formula $\mu, \nu$ can assume the values $0,1,2,3$. Afterwards we will always utilise these indices to enumerate the space dimensions only.
} 
been evaluated by F. Karsch in [0]. This point will be discussed more in detail in the section 4.2 .

The resulting one-plaquette model in this approach is then described by the following partition function:

$$
\begin{aligned}
& Z_{\mathrm{hTEK}}=\int D V \int \prod_{\mu=1}^{3} D U_{\mu} \exp \left(S_{\mathrm{hTEK}}\right) \\
& S_{\mathrm{hTEK}}=N \beta_{t}(\rho) \sum_{\mu=1}^{3} \operatorname{Re} \operatorname{Tr}\left(\mathrm{e}^{\mathrm{i} \frac{2 \pi}{L}} V U_{\mu} V^{\dagger} U_{\mu}^{\dagger}\right)+N \beta_{s}(\rho) \sum_{\mu>\nu=1}^{3} \operatorname{Re} \operatorname{Tr}\left(\mathrm{e}^{\mathrm{i} \frac{2 \pi}{L}} U_{\mu} U_{\nu} U_{\mu}^{\dagger} U_{\nu}^{\dagger}\right)
\end{aligned}
$$

where we have denoted with $V$ the link variable in the time direction, and with $U_{\mu}$ the ones in space directions. It is important to remember that only traces corresponding to closed loops in the original theoryt have physical meaning, like for instance the Polyakov loop which is given by

$$
\mathcal{P}=\frac{1}{N} \operatorname{Tr} V^{L} .
$$

We shall not consider here in detail the models based on the use of asymmetric boxes, which have the advantage of having $\beta_{s}=\beta_{t}=\beta$ but the disadvantage of far more complicated twists, at least for the space-like plaquettes. It should be noticed however that, in the approximation where space-like plaquettes are neglected, the model based on asymmetric boxes leads to the same action given in (1).

\subsection{The perturbative expansion in $\beta_{s}$}

If we were able to perform the exact integration over the space-like link variables $U_{\mu}$ in eq. (6), we would obtain the exact effective action for $V$, and thus for the Polyakov loop, in the large $N$ limit. However this is too difficult, and we shall follow a perturbative approach consisting in an expansion of the partition function (6) in powers of $\beta_{s}$. In the present paper we shall consider only the zeroth order of this expansion, namely the partition function (11) which corresponds to neglecting the space-like plaquettes altogether. An explicit calculation of the first non trivial order in $\beta_{s}$ seems to be within reach of the techniques developed in the following section, but it will be left to future investigation. The extent to which the zero order approximation is reliable was discussed in ref. [3], where the same approximation was used to study, in the large $N$ limit, the effective action for the Polyakov loop in a lattice gauge theory with both a Wilson and a heat kernel action. The difference between the approach of the present paper and the one of ref. [3]

\footnotetext{
${ }^{2}$ That is, the $d+1$-dimensional LGT (defined on a box of size $L$ ) from which the model (2) [or the model (6)] is obtained by eliminating the space-time dependence of the gauge fields via the "twisted Eguchi-Kawai" reduction (see [2], and similar arguments in next section 2.3).
} 
is that the reduction to a one plaquette model was obtained there, for the Wilson action, by an approximate Migdal-Kadanoff [8] bond-moving scheme. Instead our starting point eq. (6) is exact.

The perturbative expansion in $\beta_{s}$ was studied in even more details in ref. [4, where the expansion was carried out, for the $S U(2)$ lattice gauge theory, up to the order $\beta_{s}^{2}$. The feature that emerges from ref.s [3, 4] is that the zeroth order approximation, although it correctly predicts the existence of a deconfinement transition for large temperature, fails to reproduce the deconfinement temperature in $3+1$ dimensions for lattice sizes $n_{t}$ in the time direction greater than 2 . This is related to the fact that in the zeroth order approximation the critical value of $\beta_{t}$ rescales linearly with $n_{t}$ rather than with the logarithmic law required by asymptotic freedom. A correct prediction of the latter behaviour would presumably require an exact derivation, namely to all orders in $\beta_{s}$, of the effective action for the Polyakov loop; however in the $S U(2)$ theory the first non trivial order in $\beta_{s}$ already provides a striking improvement in the scaling properties 4 . We expect the same to happen in the large $N$ case, but in this paper we shall restrict ourselves, as already mentioned, to the zeroth order approximation, the emphasis being more on the the matrix model (11) itself and its interesting features. It should be noticed also that, unlike the $3+1$ dimensional case, in $2+1$ dimensions the full theory has the same linear rescaling with $n_{t}$ as the $\beta_{s}=0$ approximation, and that there are indications that such approximation is in that case reliable also for large $n_{t}$.

\subsection{Twisted reduction in $d+1$ dimensions. Weak coupling vacuum}

We have so far considered the reduction to a one plaquette model of finite temperature lattice gauge theory only in $3+1$ dimensions. We want now to show here that the partition function given in (1) can be obtained as the one plaquette reduction of the large $N$ finite temperature lattice gauge theory in $d+1$ dimensions, in the zeroth order approximation $\beta_{s}=0$. For even space-time dimensions we could prove this by using the existing twisted Eguchi-Kawai models, essentially in the same way as in $3+1$ dimensions. However in odd space-time dimensions consistent twists have not been constructed, and we have therefore to rely on a different type of approach.

We consider a lattice gauge theory defined on a lattice which is infinite in the space directions and of length $L$ with periodic boundary conditions in the time direction. The strategy is to reduce the height of the lattice in the time direction to one by using the same type of approach as in the Eguchi-Kawai model, and then to eliminate the dependence from space coordinates by assuming that a constant

\footnotetext{
${ }^{3}$ The lattice size $n_{t}$ introduced here corresponds to the number of links in the time direction in a lattice with the same lattice spacing in the space and time directions. So it is related to the lattice size $L$ of the Eguchi-Kawai reduction of eq.(6) by the relation $n_{t}=\frac{L}{\rho}$.
} 
master field dominates in the large $N$ limit. Let us start with the action

$$
\begin{aligned}
S(L) & =N \sum_{\vec{x}} \sum_{t=1}^{L} \operatorname{Re} \operatorname{Tr}\left(\beta_{t} \sum_{\mu=1}^{d} V(\vec{x}, t) U_{\mu}(\vec{x}, t+1) V^{\dagger}(\vec{x}+\hat{\mu}, t) U_{\mu}^{\dagger}(\vec{x}, t)\right. \\
& \left.+\beta_{s} \sum_{\mu>\nu=1}^{d} U_{\mu}(\vec{x}, t) U_{\nu}(\vec{x}+\hat{\mu}, t) U_{\mu}^{\dagger}(\vec{x}+\hat{\nu}, t) U_{\nu}^{\dagger}(\vec{x}, t)\right) .
\end{aligned}
$$

Notice that in (8) we kept two independent couplings $\beta_{s}$ and $\beta_{t}$. Unlike the case of eq. (6), however, this is not necessary in order to be able to take the limit $N \rightarrow \infty$, because $L$ and $N$ are now unrelated, and we will be free to choose $\beta_{s}=\beta_{t}$ at our convenience. The naive prescription for the reduction of the time degrees of freedom would be

$$
\begin{aligned}
V(\vec{x}, t) & \rightarrow V(\vec{x}), \\
U_{\mu}(\vec{x}, t) & \rightarrow U_{\mu}(\vec{x})
\end{aligned}
$$

It is easy to show by standard methods (see for instance [2] ) that the resulting action, which can be identified with $S(1)$, leads to the same set of loop equations in the large $N$ limit as the full $S(L)$ theory, provided all loops which are closed in the reduced lattice $(L=1)$ but correspond to open loops in the original lattice have vanishing expectation value. Just as in the untwisted Eguchi-Kawai model, this would be granted by the symmetry

$$
V(\vec{x}) \rightarrow \mathrm{e}^{i \frac{2 \pi n}{N}} V(\vec{x})
$$

which is satisfied by $S(1)$. The trace along "open" lines is not invariant under the symmetry (10) as they do not contain the same number of $V(\vec{x})$ and $V^{\dagger}(\vec{x})$ fields. So these contributions vanish unless the symmetry is broken. The symmetry however is actually broken in the weak coupling regime, that is also in the continuum limit, where $V(\vec{x})$ is close to $\mathbf{1}$ (more generically to an element of $\mathbf{Z}_{N}$ ) and the traces of open lines do not vanish. Consequently the reduction prescription must be endowed with twists, namely it must be of the type

$$
\begin{aligned}
V(\vec{x}, t) & \rightarrow D(\vec{x}, t) V(\vec{x}) D^{\dagger}(\vec{x}, t), \\
U_{\mu}(\vec{x}, t) & \rightarrow D(\vec{x}, t) U_{\mu}(\vec{x}) D^{\dagger}(\vec{x}, t)
\end{aligned}
$$

where $D(\vec{x}, t)$ is given by

$$
D(\vec{x}, t)=(\Gamma)^{\sum x_{i}}\left(\Gamma_{0}\right)^{t}
$$

with $\Gamma$ and $\Gamma_{0}$ traceless $S U(N)$ matrices satisfying the 't Hooft algebra

$$
\Gamma \Gamma_{0}=\mathrm{e}^{\mathrm{i} \frac{2 \pi}{N} m} \Gamma_{0} \Gamma
$$


In the last equation $m$ is an integer number to be determined. By performing in (8) the replacement (11) and redefining the variables according to the substitution $U_{\mu}(\vec{x}) \rightarrow U_{\mu}(\vec{x}) \Gamma$ and $V(\vec{x}) \rightarrow V(\vec{x}) \Gamma_{0}$ we obtain

$$
\begin{aligned}
Z_{R}= & \int \prod_{\vec{x}}\left[D V(\vec{x}) \prod_{\mu=1}^{d} D U_{\mu}(\vec{x})\right] \exp \left(S_{R}\right), \\
S_{R}= & \beta_{t} N \sum_{\vec{x}} \sum_{\mu=1}^{d} \operatorname{Re} \mathrm{e}^{\mathrm{i} \frac{2 \pi m}{N}} \operatorname{Tr}\left[U_{\mu}(\vec{x}) V(\vec{x}+\hat{\mu}) U_{\mu}^{\dagger}(\vec{x}) V^{\dagger}(\vec{x})\right]+ \\
& \beta_{s} N \sum_{\vec{x}} \sum_{\mu>\nu} \operatorname{Re} \operatorname{Tr}\left[U_{\mu}(\vec{x}) U_{\nu}(\vec{x}+\hat{\mu}) U_{\mu}^{\dagger}(\vec{x}+\hat{\nu}) U_{\nu}^{\dagger}(\vec{x})\right] .
\end{aligned}
$$

Notice that, unlike the twisted Eguchi-Kawai model, the twists are present in (14) only in the contributions from time-like plaquettes, as the reduction has been done only in the time direction.

Consider now the loop equations for the reduced theory (14). We already remarked that as long as the symmetry (10) is unbroken the loop equations of the reduced theory coincide with the ones of (8). We will show now that in the twisted theory this is the case also in the weak coupling regime. Indeed in the extremely weak coupling the fields tend to their vacuum configurations, which in our twisted reduced theory are

$$
\begin{aligned}
U_{i}(\vec{x}) & \rightarrow P_{\frac{N}{m}} \otimes \mathbf{1}_{m}, \\
V(\vec{x}) & \rightarrow Q_{\frac{N}{m}} \otimes \mathbf{1}_{m},
\end{aligned}
$$

where $\mathbf{1}_{m}$ is the $m \times m$ unit matrix and $P_{\frac{N}{m}}$ and $Q_{\frac{N}{m}}$ are the usual building blocks for the twist eating configurations:

$$
(P)_{a b}=\delta_{a+1, b} \quad ; \quad(Q)_{a b}=\delta_{a b} \mathrm{e}^{\mathrm{i} \frac{2 \pi m}{N} a} \quad, \quad a, b=1, \ldots N / m
$$

with periodicity in the the indices $a$ and $b$, namely $a=\frac{N}{m}+1$ means $a=1$. It is clear from the context that we have to restrict the values of $N$ so that $N / m$ is an integer. With these vacuum configurations the trace of open lines is proportional to $\operatorname{Tr}\left(Q_{\frac{N}{m}}\right)^{t}$, where $t$ is the difference between the number of $V^{\prime}$ 's and $V^{\dagger}$ 's in the trace, namely the difference between the time coordinate of the initial and the final point of the path in original unreduced lattice. It is elementary to see from eq. (16) that

$$
\operatorname{Tr}\left(Q_{\frac{N}{m}}\right)^{t}=0 \quad \text { unless } \quad t=k \frac{N}{m}
$$

for integer $k$. In the unreduced lattice the closed loops correspond to $t=k L$, so the comparison of the two equation determines $m$ :

$$
m=\frac{N}{L}
$$




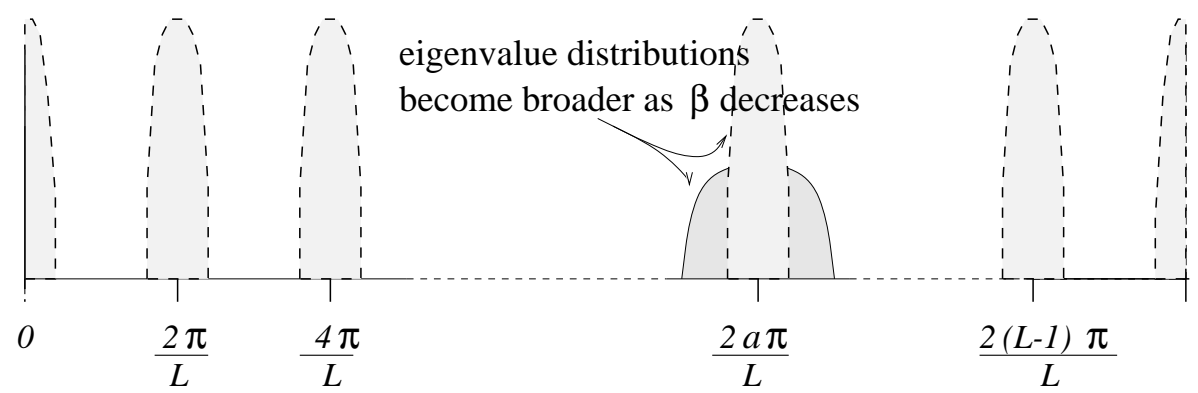

Fig. 1: The weak coupling solution in the large- $\frac{N}{L}$ consists of $L$ distributions, centered at the multiples of $\frac{2 \pi}{L}$. The width of the distributions grows as $\beta$ decreases, until finally an instability is attained at a certain critical value $\beta_{\mathrm{cr}}$.

With the above replacement eq. (14) is, in the large $N$ limit, an exact dimensional reduction of (8) on an infinite $d$-dimensional lattice. In order to obtain the matrix model (11) that we intend to study, two more steps are required. First, we assume that an $\vec{x}$-independent master field dominates the functional integral so that the field variables in (14) can be replaced by constants; second, we neglect, according to the previous discussion, the contributions of the space-like plaquettes by setting $\beta_{s}=0$; correspondingly, from now on we write simply $\beta$ for $\beta_{t}$. For $d=3$ the final result coincides with the one obtained directly from the hot twisted Eguchi-Kawai model, and the same can be shown to happen for any even space-time dimension. The derivation in the present section extends the validity of the reduction to odd space-time dimensions.

The vacuum configuration given in (15) and (16) consists for $V(\vec{x})$ of $L$ bunches of $\frac{N}{L}$ degenerate eigenvalues at the $L^{\text {th }}$ roots of 1 . This means that the open Polyakov loop $[V(\vec{x})]^{L}$ coincides in this extreme weak coupling limit with the unit matrix ${ }^{4}$. In the next section the quadratic quantum fluctuations around this vacuum are considered and their contribution is calculated, resulting into a broadening of the eigenvalue distribution within each bunch and eventually, as $\beta_{t}$ decreases, into a phase transition towards a uniform eigenvalue distribution.

\section{Derivation of the effective model}

Let us consider the partition function (1) of the twisted one-plaquette model. The integrations over the unitary matrices $U_{\mu}$ are all independent and the partition function can then be rewritten as

$$
Z=\int D V\left[\int D U \mathrm{e}^{\beta N \operatorname{Re} \mathrm{e}^{\mathrm{i} \frac{2 \pi}{L}} \operatorname{Tr}\left(V U V^{\dagger} U^{\dagger}\right)}\right]^{d}
$$

\footnotetext{
${ }^{4}$ More generically, we could in (15) replace $\mathbf{1}_{m} \equiv \mathbf{1}_{\frac{N}{L}}$ with an element of $\mathbf{Z}_{\frac{N}{L}}$. Correspondingly, $V(\vec{x})^{L}$ would be an element of $\mathbf{Z}_{N}$, which is indeed the generic situation in the broken phase.
} 


$$
=\int\left(\prod_{i=1}^{N} \mathrm{~d} \theta_{i}\right) \mathcal{J}^{2}(\theta)\left[\int D U \mathrm{e}^{\beta N \sum_{i, j}\left|U_{i j}\right|^{2} \cos \left(\theta_{i}-\theta_{j}+\frac{2 \pi}{L}\right)}\right]^{d},
$$

where in the last line we have gauge-rotated the matrix $U$ to diagonalize $V$ :

$$
V \rightarrow \operatorname{diag}\left(\mathrm{e}^{\mathrm{i} \theta_{0}}, \ldots, \mathrm{e}^{\mathrm{i} \theta_{N-1}}\right)
$$

$\mathcal{J}(\theta)$ is the Haar measure of $\mathrm{SU}(\mathrm{N})$ expressed in terms of integration over the eigenvalues $\mathrm{e}^{\mathrm{i} \theta_{i}}$ and it is given by

$$
\mathcal{J}^{2}(\theta)=\prod_{i<j} 4 \sin ^{2}\left(\frac{\theta_{i}-\theta_{j}}{2}\right)
$$

We want now to solve the model (19) in the weak coupling limit, by expanding around the vacuum configuration in which

$$
V=Q_{L} \otimes \mathbf{1}_{N / L} \quad ; \quad \mathcal{P}=\frac{1}{N} \operatorname{Tr} V^{L}=1 .
$$

In this vacuum the eigenvalues of $V$ are organised in $L$ bunches, each composed of $\frac{N}{L}$ identical values $\mathrm{e}^{\frac{2 \pi i a}{L}}, a=1, \ldots L$. Accordingly we write the eigenvalues of the matrix $V$ in the weak coupling phase as

$$
\theta_{i} \equiv \theta_{a, \alpha}=\frac{2 \pi a}{L}+\phi_{a}+\varphi_{a, \alpha}
$$

with $a=1, \ldots L, \alpha=1, \ldots \frac{N}{L}$ and with $\varphi_{a, \alpha}$ constrained by $\sum_{\alpha} \varphi_{a, \alpha}=0$. We have separated here the degrees of freedom $\phi_{a}$ corresponding to the fluctuations of the centres of the bunches from the fluctuations $\varphi_{a, \alpha}$ within each bunch.

We will show that in the large $N / L$ limit the model is solved, for $\beta$ bigger than a calculable critical value $\beta_{\mathrm{cr}}$, by an eigenvalue distribution for $V$ consisting of $\mathrm{L}$ separated distributions, all centered at a multiple of $\frac{2 \pi}{L}$. In the large $L$ limit we will show that the $L$ bunches of eigenvalues all have the same Wigner semicircular distribution.

\subsection{Integration over the space-like links}

The first step in order to solve the model is to perform explicitly the integration over the unitary matrix $U$ in the expression (19) of the partition function. This will lead us to an "effective" action for the eigenvalues of the matrix $V$, which will be studied by means of matrix models techniques.

\footnotetext{
${ }^{5}$ Here again we assume $\frac{N}{L}$ to be an integer. This assumption is justified, as in the Twisted Eguchi-Kawai model, by the belief that the large $N$ and the continuum limit (large $L$ ) are smooth. It would nevertheless be of some interest to study the case of $N=m L+r$ with $m$ and $r$ integers and $0<r<L$. Although sub-leading in the large $N$ expansion, the dynamics of the $r$ eigenvalues that cannot accommodate into $L$ identical bunches might reveal some interesting features, and it is likely to depend on the existence of common divisors of $L$ and $r$.
} 
Let us consider the slightly more general integral

$$
I(\theta, \psi)=\int D U \mathrm{e}^{\beta N \sum_{i j} \cos \left(\theta_{i}-\psi_{j}\right)\left|U_{i j}\right|^{2}},
$$

which reduces to the one of (19) for $\psi_{j}=\theta_{j}-\frac{2 \pi}{L}$.

In spite of its analogy with the Itzykson-Zuber integral, the integration in (24) cannot be done exactly (except for $N=2$ ) as the Duistermann-Heckmann theorem does not apply in this case. However in the large $N$ limit it is possible to apply to it the saddle-point method. In this way Kogan et. al [9], by taking into account, like in the Itzykson-Zuber integral, all extrema - both maxima and minima - of the exponent, obtained the following asymptotic formula:

$$
\int D U \mathrm{e}^{\sum_{i j} A_{i j}\left|U_{i j}\right|^{2}}=\sum_{P} \frac{\exp \left\{\sum_{k} A_{k P(k)}\right\}}{\prod_{i<j}\left[A_{i P(i)}+A_{j P(j)}-A_{i P(j)}-A_{j P(i)}\right]}\left(1+O\left(\frac{1}{A}\right)\right),
$$

$P$ being a permutation of the indices $\{i\}$. By replacing in (25) $A_{i j}$ with $\beta N \cos \left(\theta_{i}-\right.$ $\psi_{j}$ ) and by using the trigonometrical identity

$$
\begin{gathered}
\cos \left(\theta_{i}-\psi_{P(i)}\right)+\cos \left(\theta_{j}-\psi_{P(j)}\right)-\cos \left(\theta_{i}-\psi_{P(j)}\right)-\cos \left(\theta_{j}-\psi_{P(i)}\right) \\
=4 \sin \left(\frac{\theta_{i}-\theta_{j}}{2}\right) \sin \left(\frac{\psi_{P(i)}-\psi_{P(j)}}{2}\right) \cos \left(\frac{\theta_{i}+\theta_{j}-\psi_{P(i)}-\psi_{P(j)}}{2}\right)
\end{gathered}
$$

we would obtain the following expression for the large $N$ limit of $I(\theta, \psi)$ :

$$
I(\theta, \psi)=C \sum_{P}(-1)^{P} \mathcal{J}^{-1}(\theta) \mathcal{J}^{-1}(\psi) \frac{\exp \left\{N \beta \sum_{k} \cos \left(\theta_{k}-\psi_{P(k)}\right)\right\}}{\prod_{i, j} \cos \left(\frac{\theta_{i}-\psi_{P(i)}+\theta_{j}-\psi_{P(j)}}{2}\right)}\left(1+O\left(\frac{1}{N}\right)\right),
$$

where $C=(\beta N)^{\frac{N(N-1)}{2}}$ is an irrelevant overall factor, which will be discarded in what follows.

In applying the saddle point method to the integral at the l.h.s. of eq. (25) one should be aware that the gaussian integration around the extrema would diverge as a maximum turns into a minimum of the integrand. These divergences are signalled by the zeros at the denominator of the r.h.s of eq. (27). The zeros of $\mathcal{J}(\theta)$ and $\mathcal{J}(\psi)$ however are cancelled by the zeros at the numerator when the sum over permutations is performed, in analogy to what happens in the Itzykson-Zuber integral. Instead the zeros of $\cos \left(\frac{\theta_{i}-\psi_{P(i)}+\theta_{j}-\psi_{P(j)}}{2}\right)$ are true singularities of the r.h.s of (27). These singularities are not present in the original integral and they denote that the equation (27) cannot be applied in such circumstances unless one restricts the sum to a suitable subset of permutations.

Let us go back to our original integral of eq. (19), which is obtained from (27) with the substitution $\psi_{i} \rightarrow \theta_{i}-\frac{2 \pi}{L}$. The vacuum configuration of such a model was discussed in the previous section and it is given by eq. (15), with $N / m=L$.

To consider just quantum fluctuations around this vacuum is equivalent to restrict the sum over permutations at the r.h.s. of (27) to the permutations that map each bunch of eigenvalues into the next bunch, namely:

$$
P: \theta_{a, \alpha} \rightarrow \theta_{a+1, P_{a}(\alpha)} .
$$


where $P_{a}(\alpha)$ corresponds to a permutation of the $\frac{N}{L}$ eigenvalues of the bunch at $\frac{2 \pi a}{L}$. The other permutations are exponentially depressed compared to the ones above; in fact for each eigenvalue mapped from the bunch $a$ to the bunch $a+s+1$ the exponential in (27) is depressed by a factor

$$
\exp \left(\beta N\left(1-\cos \frac{2 \pi s}{L}\right)\right) \sim \exp \left(-\beta N \frac{2 \pi^{2} s^{2}}{L^{2}}\right) .
$$

In the next section we shall precisely examine the quantum fluctuations around the classical vacuum given in (15), and determine for what value of $\beta$ such fluctuations make the vacuum unstable. In other words we shall neglect all extrema of the action other than the ones given in eq. (28), and also keep the center of the bunches fixed at their classical value, namely we shall fix $\phi_{a}=0$ through all the calculation. Of course the question arises whether a condensation of the other extrema, or an instability in the dynamics of the bunches as a whole may cause a transition before the vacuum becomes unstable due to the quantum fluctuations. In the next section we shall give strong arguments, based on the comparison of the present model with QCD in two dimension on a cylinder, that at least for large $L$ the value of $\beta$ for which the vacuum becomes unstable in very close, if not coincident, with the one where the transition takes place. Finally, let us remark that when the sum over permutations in eq. (27) is restricted to the ones of (28) the result of the saddle point method is singularity free, as the argument of the cosine at the denominator is for those permutation close to zero.

\subsection{The effective quadratic model}

With the replacement $\psi_{i} \rightarrow \theta_{i}-\frac{2 \pi}{L}$ the r.h.s. of eq. (27) becomes

$$
\sum_{P}(-1)^{P} \mathcal{J}^{-2}(\theta) \frac{\exp \left\{N \beta \sum_{k} \cos \left(\theta_{k}-\theta_{P(k)}+\frac{2 \pi}{L}\right)\right\}}{\prod_{i, j} \cos \left(\frac{\theta_{i}-\theta_{P(i)}+\theta_{j}-\theta_{P(j)}}{2}\right)}
$$

where the sum is now restricted to the permutations given in eq. (28). We replace $\theta_{i}$ with its classical value plus fluctuations, according to eq. (23), and expand each term up to quadratic order in the fluctuations. It is easy to see that upon this expansion the fluctuations $\phi_{a}$ (the "centres") and $\varphi_{a, \alpha}$ decouple completely. As already discussed, we consider the solution where all the $\phi_{a}$ vanish. In the remaining of this section we keep therefore track only of the terms containing $\varphi_{a, \alpha}$ and we derive a quadratic effective model for them.

Let us consider first the factor $\mathcal{J}^{2}(\theta)$ appearing both in the integration volume and in (30). Under the substitution (23) it becomes

$$
\mathcal{J}^{2}(\theta)=\prod_{i j} 4 \sin ^{2} \frac{\theta_{i}-\theta_{j}}{2} \rightarrow \prod_{a, \alpha<b, \beta} 4 \sin ^{2}\left[\frac{\pi(a-b)}{L}+\frac{\varphi_{a, \alpha}-\varphi_{b, \beta}}{2}\right]
$$




$$
\begin{aligned}
=\left[\prod_{a} \Delta^{2}\left(\varphi_{a}\right)\right] \times \exp & \left\{\sum_{a \neq b} \sum_{\alpha \beta} \log \left(2 \sin \left[\frac{\pi(a-b)}{L}+\frac{\varphi_{a, \alpha}-\varphi_{b, \beta}}{2}\right]\right)\right. \\
& \left.+\sum_{a} \sum_{\alpha \neq \beta} \log \frac{\sin \frac{\varphi_{a, \alpha}-\varphi_{b, \beta}}{2}}{\frac{\varphi_{a, \alpha}-\varphi_{b, \beta}}{2}}\right\},
\end{aligned}
$$

where $\Delta^{2}\left(\varphi_{a}\right)=\prod_{\alpha<\beta}\left(\varphi_{a, \alpha}-\varphi_{a, \beta}\right)^{2}$ is the usual Cauchy-Vandermonde determinant.

The quadratic expansion of eq. (31) is

$$
\left[\prod_{a} \Delta^{2}\left(\varphi_{a}\right)\right] \times \exp \left\{-\frac{1}{4} \frac{N}{L}\left(\sum_{l=1}^{L-1} \frac{1}{\sin ^{2} \frac{\pi l}{L}}+\frac{1}{12}\right) \sum_{a, \alpha} \varphi_{a, \alpha}^{2}+O\left(\varphi^{4}\right)\right\} .
$$

The finite sum appearing above can be exactly calculated (see App. A) and it is given by

$$
\sum_{l=1}^{L-1} \frac{1}{\sin ^{2} \frac{\pi l}{L}}=\frac{L^{2}-1}{3}
$$

The exponential and the denominator in (30) become respectively

$$
\begin{aligned}
& \left.\exp \left\{\sum_{i} N \beta \sum_{k} \cos \left(\theta_{k}-\theta_{P(k)}+\frac{2 \pi}{L}\right)\right\}\right\} \rightarrow \exp \left\{\beta N \sum_{a, \alpha} \cos \left(\varphi_{a, \alpha}-\varphi_{a+1, P_{a}(\alpha)}\right)\right\} \\
& =\exp \left\{-\beta N\left(\sum_{a, \alpha} \varphi_{a, \alpha}^{2}-\sum_{a, \alpha} \varphi_{a, \alpha} \varphi_{a+1, P_{a}(\alpha)}+O\left(\varphi^{4}\right)\right)\right\}
\end{aligned}
$$

and

$$
\begin{aligned}
& \prod_{i<j}\left(\cos \frac{\theta_{i}-\theta_{P(i)}+\theta_{j}-\theta_{P(j)}}{2}\right)^{-1} \\
& \rightarrow \exp \left\{-\sum_{a, \alpha<b, \beta} \log \cos \left[\frac{\varphi_{a, \alpha}-\varphi_{a+1, P_{a}(\alpha)}+\varphi_{b, \beta}-\varphi_{b+1, P_{b}(\beta)}}{2}\right]\right\} \\
& \left.=\exp \left\{\frac{N}{4}\left(\sum_{a, \alpha} \varphi_{a, \alpha}^{2}-\sum_{a, \alpha} \varphi_{a, \alpha} \varphi_{a+1, P_{a}(\alpha)}+O\left(\varphi^{4}\right)\right)\right]\right\} .
\end{aligned}
$$

Putting all these results together, we can now write down the explicit expression of the action (19) expanded up to quadratic terms in the fluctuations $\varphi$ :

$$
\begin{gathered}
\int\left[\prod_{a \alpha} \mathrm{d} \varphi_{a, \alpha}\right]\left[\prod_{a} \Delta^{2}\left(\varphi_{a}\right)\right]^{(1-d)} \exp \left\{-\frac{N}{L}\left[\left(\beta L-\frac{L}{4}\right) d-\frac{d-1}{12} L^{2}\right] \sum_{a, \alpha} \varphi_{a, \alpha}^{2}\right\} \\
\times\left(\sum_{\left\{P_{a}\right\}}(-1)^{\sigma\left(P_{a}\right)} \exp \left\{\frac{N}{L}\left(\beta L-\frac{L}{4}\right) \sum_{a, \alpha} \varphi_{a, \alpha} \varphi_{a+1, P_{a}(\alpha)}\right\}\right)^{d} .
\end{gathered}
$$

The solution of this model is the subject of the next section. 


\section{Solution of the model}

\section{1 $\mathrm{Z}_{L}$ invariant solution}

In solving the model (36) for an arbitrary value of $L$, we assume that the master field is translational invariant, namely invariant under the $\mathbf{Z}_{L}$ symmetry of the vacuum (22). With this assumption all bunches of eigenvalues have the same distribution and we can set:

$$
\varphi_{a, \alpha} \rightarrow \frac{\varphi_{\alpha}}{L}
$$

where $\varphi_{\alpha}$ is now of order 1 in the large $L$ limit (recall that the fluctuations $\varphi_{a, \alpha}$ are of order $\frac{1}{L}$ ). The invariance under translations is at this stage an assumption, although a very reasonable one; it will be checked a posteriori in section 4.3 for an arbitrary value of $L$.

With the position (37) the partition function (36) reduces to the product of the partition functions of $L$ identical models:

$$
\begin{aligned}
Z=\left(Z_{K M}\right)^{L}= & {\left[\int \prod_{\alpha=1}^{n} \mathrm{~d} \varphi_{\alpha}\left[\Delta^{2}(\varphi)\right]^{1-d} \exp \left\{-n\left[\beta_{H} d-\frac{d-1}{12}\right] \sum_{\alpha}\left(\varphi_{\alpha}\right)^{2}\right\}\right.} \\
& \left.\times\left(\sum_{P}(-1)^{\sigma(P)} \exp \left\{n \beta_{H} \sum_{\alpha} \varphi_{\alpha} \varphi_{P_{a}(\alpha)}\right\}\right)^{d}\right]^{L}
\end{aligned}
$$

where we have defined

$$
\beta_{H}=\frac{1}{L}\left(\beta-\frac{1}{4}\right)
$$

and $n=N / L$. The partition function of each individual model in the above equation coincides with the one of a $d$-dimensional Kazakov-Migdal model [10] with quadratic potential. In fact from the Kazakov-Migdal partition function

$$
\begin{array}{r}
\int \prod_{\mu=1}^{d} \prod_{\vec{x}} D U_{\mu}(\vec{x}) \int \prod_{\vec{x}} D \Phi(\vec{x}) \exp \left\{-n \beta_{H} \operatorname{Tr} \sum_{\vec{x}}\left[\frac{m^{2}}{2} \Phi^{2}(\vec{x})\right.\right. \\
\left.\left.-\sum_{\mu=1}^{d} \Phi(\vec{x}) U_{\mu}(\vec{x}) \Phi(\vec{x}+\mu) U_{\mu}^{\dagger}(\vec{x})\right]\right\}
\end{array}
$$

by integrating over the unitary matrices $U_{\mu}(\vec{x})$ one obtains in the mean-field approximation $(\Phi(\vec{x})$ independent of $\vec{x})$ the expression $Z_{K M}$ in (38), where $\left\{\varphi_{\alpha}\right\}$ are the eigenvalues of the hermitean matrix $\Phi$, and the mass $m$ is:

$$
m^{2}=2 d-\frac{d-1}{6 \beta_{H}}
$$

The model (40) was solved in the large- $n$ limit by Gross [1], who showed that the 
eigenvalue distribution $\rho(\varphi)$ is semicircular:

$$
\begin{array}{ll}
\rho(\varphi)=\frac{2}{\pi r^{2}} \sqrt{r^{2}-\varphi^{2}} & \text { for }|\varphi|<r, \\
\rho(\varphi)=0 & \text { for } r<|\varphi|<\pi
\end{array}
$$

with radius given by

$$
r^{2}=\frac{4(2 d-1)}{\beta_{H}\left(m^{2}(d-1)+d \sqrt{m^{4}-4(2 d-1)}\right)} .
$$

The solution becomes unstable at the value of $\beta_{H}$ for which the radius become complex, namely

$$
\beta_{H}=\frac{d-1}{12(d-\sqrt{2 d-1})} .
$$

The corresponding value of $\beta$ (recall the definition (39)) represents a lower bound for the true critical coupling $\beta_{\text {cr }}$ at which a phase transition takes place.

It should be noticed however that a different upper limit may be found by looking at the value of $\beta_{H}$ for which the radius of the eigenvalue distribution of $\varphi_{\alpha}$ becomes $\pi$, corresponding to a radius $\frac{\pi}{L}$ for each bunch of eigenvalues. A phase transition occurs at this point as the bunches merge together and the semicircular distribution is not anymore a solution.

A better understanding of the phase transition can be obtained by noticing that for large $L$, namely in the continuum limit, the Wilson action becomes equivalent to the heat-kernel action [13, 14]. To be more precise, if we consider the action (8) with $\beta_{s}=0$, in the large $L$ and hence large $\beta_{t}$ limit we have

$$
\begin{aligned}
& \exp \left(N \beta \sum_{\vec{x}} \sum_{t=1}^{L} \sum_{\mu=1}^{d} \operatorname{Re} \operatorname{Tr} U_{\mu}(\vec{x}, t) V(\vec{x}+\hat{\mu}, t) U_{\mu}^{\dagger}(\vec{x}, t+1) V^{\dagger}(\vec{x}, t)\right) \stackrel{\beta \rightarrow \infty}{\longrightarrow} \\
& \prod_{\vec{x}, t, \mu}\left\{\sum_{r} d_{r} \chi_{r}\left(U_{\mu}(\vec{x}, t) V(\vec{x}+\hat{\mu}, t) U_{\mu}^{\dagger}(\vec{x}, t+1) V^{\dagger}(\vec{x}, t)\right) \exp \left(-\frac{C_{r}}{2 N \beta}\right)\right\},
\end{aligned}
$$

where $r$ labels the irreducible representations of $S U(N), d_{r}$ their dimensions, $C_{r}$ their quadratic Casimir and $\chi_{r}(U)$ the corresponding character of $U$. Consistently with previous notations we denote $\beta_{t}$ by $\beta$ in (45) and following equations. The heat-kernel action at the r.h.s. of (45) was studied in detail in ref.s [3] and [12], which papers we refer the reader to for further details. The integration over the space-like links can be done exactly by using well known properties of characters, leading to the following action for the Polyakov loop:

$$
\exp \left(S_{\mathrm{Pol}}\right)=\prod_{\vec{x}, \mu} \sum_{r} \chi_{r}(\mathcal{P}(\vec{x}+\hat{\mu})) \chi_{r}\left(\mathcal{P}^{\dagger}(\vec{x})\right) \exp \left(-\frac{C_{r} L}{2 N \beta}\right)
$$


where $\mathcal{P}(\vec{x})=\prod_{t=1}^{L} V(\vec{x}, t)$ is the untraced Polyakov loop. By assuming as usual that the master field is invariant under translations, it was shown in [3] that from (46) one obtains the following partition function:

$$
\begin{aligned}
Z= & \int \prod_{i=1}^{N} \mathrm{~d} \hat{\theta}_{i}\left[\Delta^{2}(\hat{\theta})\right]^{1-d} \exp \left\{-N\left[\frac{\beta}{L} d-\frac{d-1}{12}\right] \sum_{i}\left(\hat{\theta}_{i}\right)^{2}\right\} \\
& \times\left[\sum_{p}(-1)^{\sigma(P)} \exp \left\{N \frac{\beta}{L} \hat{\theta}_{i} \hat{\theta}_{P i}\right\}\right]^{d} .
\end{aligned}
$$

The integration variables $\hat{\theta}_{i}$ in eq. (47) are the invariant angles of the Polyakov loop, and higher order terms in $\hat{\theta}_{i}$ in the exponent have been neglected. The partition function given in (47) is again a Kazakov-Migdal model with a quadratic potential, and it admits the same semicircular distribution (42) as the partition function (38) obtained from our one plaquette model, but with $\beta_{H}$ replaced by $\beta / L$. This is in complete agreement with the leading order in $L$ of the rescaling law given by eq. (39). The only approximation involved in deriving eq. (47) is the truncation of the power expansion in $\hat{\theta}_{i}$ at the exponent to the quadratic terms. In conclusion, the twisted one plaquette model and the heat-kernel model have in the large $L$ limit the same solution for the eigenvalue distribution of the Polyakov loop, and the same critical value of the coupling constant. This confirms that the local extrema which were neglected in the saddle point evaluation of (24) are indeed irrelevant, at least in the large $L$ limit and up to the critical value of the coupling constant.

The relation between our one plaquette model and the heat-kernel model discussed in [3] also provides a new and a priori independent way of estimating the critical coupling. In fact it was shown in [3], and it can be recognised in (46)], that in the heat-kernel model the building block of the effective action for the Polyakov loop is given by the action of QCD2 on a cylinder of area inversely proportional to $\beta / L$. So the critical value of $\beta_{H} \sim \beta / L$ should correspond to the one where the Douglas-Kazakov phase transition [15] occurs. The point of the Douglas-Kazakov phase transition is known exactly as a function of the area of the cylinder and of the radius of the semicircular distributions at its boundaries. The radius of the distribution is obtained from the solution of (47) and it is given, as before, by (43). The critical coupling is determined now by the radius where the Douglas-Kazakov phase transition occurs (see eqs. (33) and (42) in [3]) rather than by looking at the point where the radius becomes complex. The results of Table I show that all the above methods give, at least for low $d$, values for the critical $\beta_{H}$ which are very close to each other, and that the ones obtained from the analysis of the Douglas-Kazakov phase transition give consistently a better (higher) lower limit.

It is well known that the Douglas-Kazakov phase transition is due to the condensation of "instanton" contributions, namely to configurations where the initial

\footnotetext{
${ }^{6}$ The expression under the product sign in (46) is the partition function for QCD2 on on a cylinder with boundary conditions given by the Polyakov loops in $\vec{x}$ and $\vec{x}+\hat{\mu}$.
} 
Table I: Critical value of $\beta_{H}$ calculated in different ways: (a) limiting value for real radius, (b) limiting value for $r<\pi$ and (c) value corresponding to the DK phase transition in QCD2 [3]. For $d>3$ the solution becomes unstable before the radius reaches the value $\pi$.

\begin{tabular}{cccc}
\hline & (a) & (b) & (c) \\
\hline$d=2$ & 0.311 & 0.314 & 0.321 \\
$d=3$ & 0.218 & 0.218 & 0.226 \\
$d=4$ & 0.184 & $*$ & 0.192 \\
$d=5$ & 0.167 & $*$ & 0.173 \\
\hline \hline
\end{tabular}

and final value of one or more eigenvalues of $\mathcal{P}$ differ of more than $2 \pi$. We argue that there is a precise correspondence between the instantons in QCD2 on a cylinder and the classical solutions of the one plaquette model (extrema of the integral $\left.I\left(\theta, \theta-\frac{2 \pi}{L}\right)\right)$ which were neglected in the saddle point calculation of the previous section. These correspond to permutations which are not of the type of eq. (28), because one or more eigenvalues of $V$ are not mapped from a bunch $a$ to $a+1$. The argument goes as follows: in the large $L$ limit the eigenvalue distribution of our solution can be represented on an infinite line as a sequence of bunches, each made of $n=N / L$ eigenvalues $]$, at intervals of $2 \pi$. Above the phase transition different bunches do not communicate with each other, except for the canonical shift of 1 due to the twist, and the partition function is just the one corresponding to a single bunch raised to the power $L$ (see eq. (38)). The picture is quite similar for the eigenvalues of the Polyakov loop in the heat-kernel model. Their distribution can be represented on an infinite line as a periodic distribution of period $2 \pi$, that before the phase transition consists of separate bunches of $N$ eigenvalues. Let us compare now the contribution of classical solutions of the instanton type in the two models. Instanton contributions to the partition function of QCD2 on a cylinder can be calculated from the following equation (see eq. (8) in [16])

$$
\begin{aligned}
& \sum_{r} \chi_{r}(-\hat{\theta}) \chi_{r}(\hat{\varphi}) \exp \left(-\frac{t C_{r}}{2}\right) \\
\propto & \sum_{P} \frac{t^{(1-N) / 2}}{\mathcal{J}(\hat{\theta}) \mathcal{J}(\hat{\varphi})} \sum_{\left\{s_{i}\right\}} \exp \left[-\frac{1}{2 t} \sum_{i=1}^{N}\left(\hat{\varphi}_{i}-\hat{\theta}_{P(i)}+2 \pi s_{i}\right)^{2}\right] .
\end{aligned}
$$

where $t$ can be identified with $\frac{L}{N \beta}$, by comparing eq. (48) with (46). The integers $s_{i}$ correspond to the winding numbers of the eigenvalues, so the contribution of an instanton with winding number $s$ is proportional to $\exp \left(-2 \pi^{2} \beta N s^{2} / L\right)$.

\footnotetext{
${ }^{7}$ We refer here to the eigenvalues normalised as $\varphi_{\alpha}$ in 37 .
} 
Let us compare this result with the non perturbative contribution (29) corresponding to an eigenvalue being mapped by a permutation $P$ from a bunch $a$ to the bunch $a+s+1$ in the twisted one plaquette model. The r.h.s. of (29) coincide with the instanton contribution calculated above, if one takes into account that the number of eigenvalues is here $n=N / L$ instead of $N$.

We can conclude that the mechanism underlying the phase transition is the same in QCD2 on a cylinder and in the twisted one plaquette model. In particular in the latter model only fluctuations around the twist eating configurations need to be considered for $\beta$ larger than its critical value; when the critical value is attained a condensation of local extrema of the action takes place, that is responsible for the phase transition studied in this section.

\subsection{Quantum rescaling}

Let us consider again the rescaling law given by eq. (39), and write it as:

$$
\beta(L)=L \beta_{H}+\frac{1}{4}
$$

where in the 1.h.s. we replaced $\beta$ with $\beta(L)$ to remark the fact that the coupling refers to a model with $L$ time-like links. On the other hand we have $L=\rho n_{t}$ (see footnote in section 2.2), where $\rho$ is the asymmetry parameter and $n_{t}$ the number of links in the time direction in the equivalent symmetric lattice. By writing eq. (49) with $L=\rho n_{t}$ and with $L=n_{t}$, and by eliminating $\beta_{H}$ from the two equations, we find

$$
\beta\left(\rho n_{t}\right)=\rho\left(\beta\left(n_{t}\right)-\frac{1}{4}\right)+\frac{1}{4} .
$$

This equation gives the riscaling of the coupling constant induced by varying the asymmetry parameter $\rho$, and it should be compared with the first of eqs. (5) where $\beta_{\text {symm }} \rightarrow \beta\left(n_{t}\right)$ and $\beta_{t}(\rho) \rightarrow \beta\left(\rho n_{t}\right)$. The function $c_{\tau}(\rho)$ of eq. (5) was calculated by Karsch in [5]. Its behaviour for large $\rho$ is of the type

$$
4 \rho c_{\tau}(\rho)=\alpha_{t}^{0} \rho+\alpha_{t}^{1}+O\left(\frac{1}{\rho}\right)
$$

where the values of the coefficients $\alpha_{t}^{0}$ and $\alpha_{t}^{1}$ in the large- $N$ limit can be calculated from the eq. 2.25 of $[5]$ :

$$
\alpha_{t}^{0}=-0.2609 \quad ; \quad \alpha_{t}^{1}=\frac{1}{4} .
$$

We can extract for comparison the same quantities from the rescaling (50), following from our effective model. We find :

$$
\alpha_{t}^{0}=-\frac{1}{4} \quad ; \quad \alpha_{t}^{1}=\frac{1}{4}
$$


The agreement is quite remarkable, considering that Karsch's calculation took into account the space-like plaquettes, and it confirms a feature that already emerged in the case of $\mathrm{SU}(2)$ [4], namely that the corrections to $c_{\tau}(\rho)$ due to the space-like plaquettes are relatively small. The agreement between (53) and (52) also represents a further check that only the fluctuations around the classical vacuum are relevant in the deconfined phase.

\subsection{Evidence for unbroken $\mathrm{Z}_{L}$ symmetry}

In section 4 we solved the model described by the action (36) by assuming that the solution is translational invariant in the one dimensional space labelled by the index $a$ in $\varphi_{a, \alpha}$. In other words we assumed that the $Z_{L}$ symmetry of the vacuum (22) is not broken by the quantum fluctuations. Although this is justified in the large $L$ limit, it would be desirable to have an independent proof valid for all values of $L$. In this section we shall prove that for any $L$ the partition function (36) becomes singular at the critical value of $\beta$ derived in the previous section, namely at the value of $\beta$ for which the radius of the large $\mathrm{L}$ semicircular solution becomes complex.

To begin with, let us notice that the partition function (36) can be obtained by integration [17] over the unitary matrices $U$ of the following model of KazakovMigdal type:

$$
Z=\int\left[\prod_{\mu=1}^{d} D U_{\mu}^{(a)}\right]\left[\prod_{a=1}^{L} D \Phi_{a}\right] \exp \left\{-n \beta_{H} \operatorname{Tr}\left[\sum_{a} \frac{m^{2}}{2} \Phi_{a}^{2}-\sum_{a, \mu} \Phi_{a} U_{\mu}^{(a)} \Phi_{a+1} U_{\mu}^{(a) \dagger}\right]\right\},
$$

where the matrices $\Phi_{a}$ are normalised so that their eigenvalues are $L \varphi_{a, \alpha}$, and the parameters $\beta_{H}$ and $m^{2}$ are given by eqs. (39) and (41).

The structure of the model (54) is illustrated in Fig. 2; it looks like a KM model defined on a compact one-dimensional lattice of length $L$, but with $d$ links joining two neighbouring sites.

If one integrates eq. (54) over the hermitean matrices $\Phi_{a}$ rather than over the unitary matrices $U$, one obtains the "induced gauge model" [10]

$$
Z=\int\left[\prod_{a=0}^{L-1} \prod_{\mu=1}^{d} D U_{\mu}^{(a)}\right] \exp \left\{\frac{1}{2} \sum_{\Gamma} \frac{\left|\operatorname{Tr} U_{\Gamma}\right|^{2}}{l_{\Gamma}\left(m^{2}\right)^{l_{\Gamma}}}\right\}
$$

where $\Gamma$ is a closed path on the lattice of Fig. 2 and $l_{\Gamma}$ its length. $U_{\Gamma}$ is the ordered product of the link matrices $U_{\mu}^{(a)}$ on the path $\Gamma$.

Consider now the quantity $\rho_{a}^{2}=\frac{1}{n}\left\langle\operatorname{Tr} \Phi_{a}^{2}\right\rangle$, which is a measure of the width of the $a^{\prime}$ th bunch and is proportional, in case of a semicircular distribution, to the squared radius $r^{2}$. It is easy to show, by using eq. (54), that

$$
\rho_{a}^{2}=\frac{1}{n}\left\langle\operatorname{Tr} \Phi_{a}^{2}\right\rangle=\frac{1}{\beta_{H}}\left\langle\sum_{\Gamma} \frac{\left|\operatorname{Tr} U_{\Gamma}\right|^{2}}{n^{2}\left(m^{2}\right)^{l_{\Gamma}+1}}\right\rangle,
$$




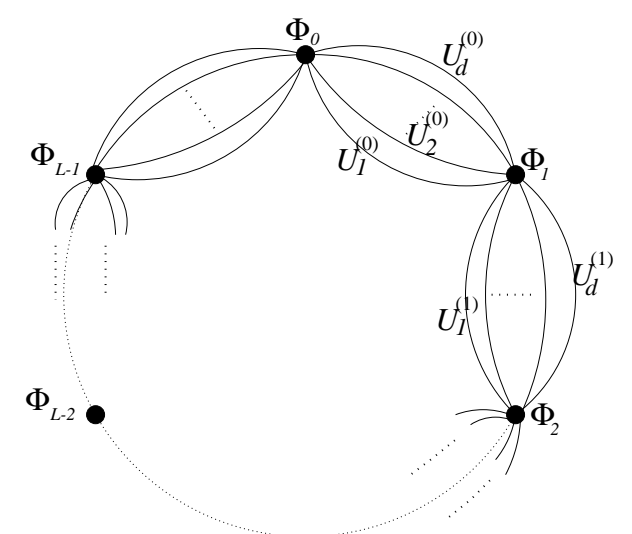

Fig. 2: Structure of the effective KM model corresponding to the case of $L$ generic. Each link $\stackrel{\Phi_{a}}{\bullet} \stackrel{U_{\mu}^{(a)}}{\longrightarrow} \Phi_{a+1}$ represents a term $\Phi_{a} U_{\mu}^{(a)} \Phi_{a+1} U_{\mu}^{(a) \dagger}$ in the action.

where the sum is over all paths $\Gamma$ beginning and ending in $a$ and the last expectation value is taken with respect to the partition function (55). In the large- $n$ limit we can use the factorisation property: $\left\langle\left|\operatorname{Tr} U_{\Gamma}\right|^{2}\right\rangle=\left\langle\operatorname{Tr} U_{\Gamma}\right\rangle\left\langle\operatorname{Tr} U_{\Gamma}^{\dagger}\right\rangle$. Moreover due to the following symmetry of the action (55):

$$
U_{\mu}^{(a)} \rightarrow \zeta_{\mu}^{(a)} U_{\mu}^{(a)}, \quad \zeta_{\mu}^{(a)} \in \mathbf{Z}_{n}
$$

which is unbroken in the weak coupling (large $\beta_{H}$ ) regime, we have:

$$
\left\langle\operatorname{Tr} U_{\Gamma}\right\rangle= \begin{cases}0 & \text { if } \Gamma \text { is not a "tree" } \\ \operatorname{Tr} \mathbf{1}=n & \text { if } \Gamma \text { is a "tree" }\end{cases}
$$

where by "tree" we denote any closed path $\Gamma$ that contains $U_{\mu}^{(a)}$ and $U_{\mu}^{(a) \dagger}$ the same number of times for any $\mu$ and $a$. By using this property, eq. (56) reduces simply to

$$
\rho_{a}^{2}=\frac{1}{\beta_{H}} \sum_{l} \frac{n(l)}{\left(m^{2}\right)^{l+1}},
$$

where $n(l)$ is the number of trees of fixed length $l$ on the lattice of Fig. 2 starting end ending in the point $a$. The instability of the weak coupling configuration occurs at the value of $m^{2}$ such that the series at the r.h.s. of eq. (59) diverges.

The basic point now is that 8 , irrespectively of the choice of the lattice on which the Kazakov-Migdal model (with quadratic potential) is defined, the expectation value of $\left\langle\operatorname{Tr} \Phi^{2}\right\rangle$ on a site of the lattice is given by $(59)$ where $n(l)$ is the number of trees of fixed length $l$ on the lattice we considered, starting end ending in the chosen point. On the other hand, $n(l)$ depends only from the coordination number of the

\footnotetext{
${ }^{8}$ For related discussions, see [19].
} 
lattice, and hence it is the same for the lattice of Fig. 2 and for a $d$-dimensional hypercubic lattice. In the latter case we know the exact solution, a semicircular distribution of radius given by 43 ); $\left\langle\operatorname{Tr} \Phi^{2}\right\rangle$ is proportional to the square of such radius and it becomes singular at the value of $\beta_{H}$ given in eq. (44). In conclusion, although we have not been able to solve explicitly for all $L$ 's the model (36) without making the explicit assumption that the $Z_{L}$ symmetry of the vacuum is unbroken, we have proved that (36) becomes singular at exactly the same critical value of $\beta_{H}$ as our $Z_{L}$ invariant solution. This shows that no phase transition associated to the breaking of the $Z_{L}$ symmetry occurs and it is a conclusive evidence that the $Z_{L}$ invariance of the vacuum is preserved.

\section{Conclusions}

We have considered the one plaquette matrix model corresponding to a hot twisted Eguchi-Kawai model in which the time-like plaquettes are neglected, and we have found a solution by calculating the quadratic quantum fluctuations around the classical vacuum, namely around the twist eating configurations. The solution for the time-like link variables consists of $L$ bunches of eigenvalues, centered around the $L^{\text {th }}$ roots of unity, each bunch with a semicircular distribution of eigenvalues. This eigenvalue distribution, although rather unusual, does not come as a surprise in this context because it corresponds to a spreading of the eigenvalue distribution of the classical vacuum of the Twisted Eguchi-Kawai model. Our solution becomes unstable at a critical value of the coupling constant, where a phase transition occurs. The crucial point here is that our calculation neglects the contributions of the other extrema of the action, so that we do not know, without further information, the range of validity of the solution. This problem is solved by observing that in the hamiltonian limit $(L \rightarrow \infty)$ the original model is mapped into one with a heat-kernel action, which was solved in a previous paper [3]. As the two solution coincide we can safely say that the contribution from the relative extrema of the action can be neglected until the instability sets in. We can actually infer that the instability of the solution is determined by a condensation of the local extrema of the action. In fact it was shown in [3] that the phase transition in the heat-kernel action is mathematically the same as the Douglas-Kazakov phase transition in QCD2, which is known to be due to a condensation of classical solutions (instantons). An interesting possibility is that the full theory, namely the one including the space-like plaquettes, might follow exactly the same pattern. It has already been suggested [20] that a condensation of the extrema of the classical action is responsible for confinement in the Twisted Eguchi-Kawai model; the results of the present paper suggest that the fluctuations around the twist eating configurations are the only ones that need to be taken into account above the deconfinement transition and that they determine the master field of the theory in that phase. An instability of the corresponding solution would then give a lower bound for the critical coupling, 
without having to take into account explicitly the complicated structure of the local extrema, whose condensation is ultimately responsible of the deconfinement transition at the critical temperature. The comparison between column (a) and (c) of Table I shows that the instability of the solution in the deconfined phase and the condensation of "instanton" solutions are indeed very close to each other, at least in our simplified model.

\section{Acknowledgements}

We thank M. Caselle and S. Panzeri for many helpful discussions. This work was supported in part by EEC Science program SC1*CT92-0789.

\section{A Appendix}

In this Appendix we derive the summation formula given in eq. (33), which is crucial in the computation of the quadratic effective model of section 3 .

This formula can be derived starting from the identity

$$
\prod_{l=0}^{L-1} 2 \sin \left(x+\frac{\pi l}{L}\right)=2 \sin L x
$$

which in turn can be proved by expanding the sine functions in exponentials and by noticing that the $L^{\text {th }}$ roots of unity satisfy the property:

$$
\sum_{k=0}^{L-1} \mathrm{e}^{-\frac{2 \pi \mathrm{i}}{L} k n}=0, \quad n=1,2, \ldots, L-1 .
$$

By taking the logarithm of both sides of eq. (60) one obtains the relation:

$$
\sum_{l=1}^{L-1} \log \left[2 \sin \left(x+\frac{\pi l}{L}\right)\right]=\log \frac{\sin L x}{\sin x},
$$

which is the "generating identity" of a set of non-trivial trigonometric summation formulas. In fact by expanding the two sides of eq. (62) in powers of $x$ we have:

$$
\begin{array}{rrr}
O(x): & \sum_{l=1}^{L-1} \cot \frac{\pi l}{L} & =0, \\
O\left(x^{2}\right): & \sum_{l=1}^{L-1} \sin ^{-2} \frac{\pi l}{L} & =\frac{L^{2}-1}{3},
\end{array}
$$

where the last equation coincides with (33). It is worth to remark here that, by taking appropriate combinations of higher order identities, one can evaluate from the power expansion of (62) all the sums of the form $\sum_{l=1}^{L-1} \sin ^{-2 k} \frac{\pi l}{L}$. For instance, form the fourth and sixth order in $x$ one gets:

$$
\sum_{l=1}^{L-1} \sin ^{-4} \frac{\pi l}{L}=\frac{\left(L^{4}-1\right)}{45}+\frac{2\left(L^{2}-1\right)}{9}
$$


and

$$
\sum_{l=1}^{L-1} \sin ^{-6} \frac{\pi l}{L}=\frac{2\left(L^{6}-1\right)}{945}+\frac{\left(L^{4}-1\right)}{45}+\frac{8\left(L^{2}-1\right)}{45}
$$

\section{References}

[1] T. Eguchi and H. Kawai, Phys. Rev. Lett. 48 (1982) 1063.

[2] For a review on Eguchi-Kawai models see S.R. Das, Rev. of Modern Physics 59 (1987) 235 and references therein.

[3] M. Billó, M. Caselle, A. D’Adda, L. Magnea and S. Panzeri, Nucl. Phys. B435 (1995) 172.

[4] M. Billó, M. Caselle, A. D’Adda, and S. Panzeri, Nucl. Phys. B472 (1996) 163.

[5] F. Karsch, Nucl. Phys. B205 (1982) 285.

[6] F. Klinkhammer and P. van Baal, Nucl. Phys. B237 (1984) 274.

[7] K. Fabricius and C. P. Korthals-Altes, Nucl. Phys. B240 (1984) 237.

[8] A. Migdal, Sov. Phys. JETP 42 (1976) 413, 743. L.P. Kadanoff, Ann. Phys. (N.Y.) 100, (1976) 359, and Rev. Mod. Phys. 49 (1977) 267.

[9] I.I. Kogan et al., Nucl. Phys. B395 (1993) 547.

[10] V.A. Kazakov and A.A. Migdal, Nucl. Phys. B397 (1993) 214.

[11] D. Gross, Phys. Lett. B293 (1992) 181.

[12] K. Zarembo, Mod. Phys. Lett. A10 (1995) 677.

[13] J.M.Drouffe, Phys. Rev. D18 (1978) 1174.

[14] P. Menotti and E. Onofri, Nucl. Phys. B190 (1981) 288.

[15] M.R. Douglas and V.A. Kazakov, Phys. Lett. B319 (1993) 219.

[16] M. Caselle, A. D’Adda, L. Magnea and S. Panzeri, hep-th/9309107, Proc. Trieste Summer School in high energy physics and cosmology, World Scientific; D.Gross and A. Matytsin, Nucl. Phys. B437 (1994) 541.

[17] Harish-Chandra, Am. I. Math. 79 (1957) 87; C. Itzykson and J.B. Zuber, J. Math. Phys. 21 (1980) 411; M.L. Mehta, Comm. Math. Phys 79 (1981) 327.

[18] M. Caselle, A. D'Adda and S. Panzeri, Phys. Lett. B293 (1992) 161. 
[19] Y. Makeenko and H.W. Pe, ITEP-TH-13/95, hep-th/9601139; Y. Makeenko, Mod. Phys. Lett A8, No. 3 (1993) 209.

[20] P. van Baal, Comm. Math. Phys. 9 (1983) 1. 